\title{
The Design of Multirotor Aircraft-based Environmental Detection System
}

\author{
Maoyan Fan ${ }^{1}$, Lifang Zhang ${ }^{2, a}$ \\ ${ }^{1}$ School of Physicsand Electronic Engineering, Yuxi Normal University, Yuxi 653100, Yunan Province, China \\ ${ }^{2}$ Office of Academic Affairs, Yuxi Normal University, Yuxi 653100, Yunan Province, China
}

\begin{abstract}
This research aims at an environmental parameter detection system based on the small quadrotor UAV. The system comprises a quadrotor UAV platform, an environmental parameter detection system, a data acquisition system and a data processing system. The temperature and humidity, as well as the concentrations of particulate matters, carbon monoxide, carbon dioxide and sulfur dioxide are detected by various types of sensors, and the data are read, written and stored by Micro SD card module. Through validation, it is found that the system has the advantages of low cost, easy operation, flexibility and convenience and can expand other types of sensors to meet different environmental information measurement requirements and reflect the environmental information in a real-time manner.
\end{abstract}

\section{Introduction}

These The Law on the Prevention and Control of Atmospheric Pollution, which is effective since the beginning of 2016, reflects the serious concern and determination of China to thoroughly control the problem of atmospheric pollution and emphasizes that regulatory measures and detection technologies can lay a basis for and guarantee the prevention and control of atmospheric pollution. The atmospheric air quality detection system with an unmanned aerial vehicle (UAV) as the flight platform has the advantages of wide detection level, quick data acquisition and small topographic interference, which can effectively make up deficiencies of the traditional detection method. This is one development direction for future environmental detection system ${ }^{[1]}$.

The economic growth of China in recent years has improved the quality of life, but also causes some damage to the surrounding environment. The environmental monitoring system started late in China, but develops very quickly over several years. With the environmental detection base stations and mobile detection vehicles, a complete monitoring system has been constructed ${ }^{[2,3]}$. However, it is impossible to detect some remote regions or those suffered from disaster and thus resulting in a series of problems. In these years, the technologies of UAV have been rapidly developed. The payload can carry small portable environmental monitor instrument, but this technology has been applied in a large scale in the field of environmental detection ${ }^{[4]}$. In this paper, a system is designed with the integration of quadrotor UAV and environmental detection, so that it is easy to monitor the surrounding air quality and reduce the workload of the environmental protection personnel.

\section{Aerodynamics and Dynamics Model of Quadrotor Aerial Vehicles}

The multirotor flight dynamics model derives from the flight dynamics model of the utility helicopter DelCopter of the Delft University of Technology. Based on the conventional analytical model of the blade-element theory, the rotor aerodynamics integrate forces on each of blade elements to obtain 3 components of forces and 3 components of moments of a single rotor in the coordinate system coinciding with the rotor shaft ${ }^{[5-6]}$. It is assumed that the rotor has no setting angle, that is, the rotor shaft is parallel to Z-axis of the airframe, so we can obtain the total aerodynamic forces $\left[X_{r} ; Y_{r} ; Z_{r}\right]$ and the total aerodynamic moment $\left[X_{r} ; Y_{r} ; Z_{r}\right]$ of all rotors.

$\left[\begin{array}{l}X_{r} \\ Y_{r} \\ Z_{r}\end{array}\right]=\sum_{i=1}^{N_{r}}\left[\begin{array}{l}X_{i} \\ Y_{i} \\ Z_{i}\end{array}\right]\left[\begin{array}{c}L_{r} \\ M_{r} \\ N_{r}\end{array}\right]=\sum_{i=1}^{N_{r}}\left(\left[\begin{array}{c}x_{i} \\ y_{i} \\ z_{i}\end{array}\right] \times\left[\begin{array}{c}X_{i} \\ Y_{i} \\ Z_{i}\end{array}\right]+\left[\begin{array}{c}L_{i} \\ M_{i} \\ N_{i}\end{array}\right]\right)$

Here: $x_{i} 、 y_{i}$ and $z_{i}$ denote positions of the rotors in the airframe coordinate system; $N_{i}$ represents the number of rotors. For the fuselage aerodynamics, we suppose that the fuselage produces only a drag force $D_{f}$ along the direction of inflow and that the aerodynamic center coincides with the center of gravity, that is to say, no moment generated at the center of gravity:

$$
D_{f}=\frac{1}{2} \rho F_{0}\left(\mu^{2}+v^{2}+\omega^{2}\right)
$$


Here: $\rho$ is the air density; $F_{0}$ is the fuselage drag constant; $\mu, v$ and $\omega$ are the linear velocities in the directions of $x, y$ and $z$ axes of the airframe. In order to simplify the analysis, the simplified motor model is used. Suppose that the motor power output can follow the input signal in a real-time manner, the motor can be obtained according to the power and speed:

$$
Q_{i}=\frac{P_{\max } U_{i}}{\Omega_{i}}
$$

Here: $Q_{i}$ is the torque of the $i$-th motor; $P_{\max }$ is the maximum motor power; $U_{i}$ is the dimensionless input signal of the $i$-th motor; $\Omega_{i}$ is the current speed. Integrated with the rotor aerodynamic forces, the rotor and the motor speed variation are:

$$
\dot{\Omega}_{i}=\frac{Q_{i}-N_{i}}{I_{r}+I_{m}}
$$

Here: $I_{r}$ and $I_{m}$ are the moment of inertias of the rotor and the motor, respectively. Substitute the aerodynamic forces determined by Eqs.(1) and (3) into the conventional equation of motion for a rigid body of aircraft, we can obtain the airframe aerodynamic portion of the multirotor flight dynamics model: $\dot{X}_{b}=E_{b}\left(X_{b}, \Omega\right)$, where $E_{b}$ represents the nonlinear equations of the airframe motion; $X_{b}$ denotes the degree of freedom (DOF) of rigid body motion of the airframe, which comprises 3 translational velocities, 3 rotating angular velocities and 3 Euler angles; $\Omega$ is the DOF of rotor speed, including the rotating angular velocities of all rotors. The quadrotor UAV has no tail structure, so that the entire airframe becomes compact and the lift forces generated on four rotor arms are more uniform than a single motor, so the aerial vehicle flies more stable. The schematic diagram of the structural model of the quad-rotor UAV is shown in Fig.1. Four rotor arms in the "cross" or "X" shape are often fixed on the quadrotor center plate. One motor is installed on each rotor arm. The flight attitude can be controlled through the control of motor speed during flight.

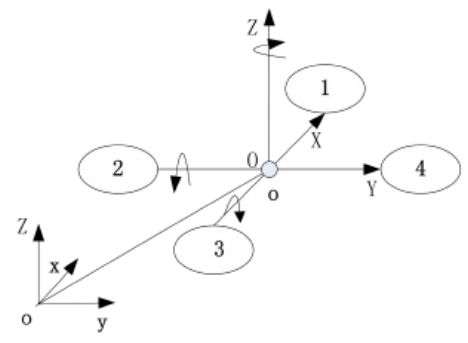

Fig.1 Quad-rotor coordinate system schematic diagram

In practice, the quadrotor UAV is a complex system. The mathematical model should be simplified for better modeling. We suppose that the whole airframe is a homogeneous symmetrical rigid body that does not deform, the multirotor aircraft has quite different characteristics of mode from those of traditional helicopters. The multirotor aircraft modes have the following characteristics: (1) due to the symmetry in the vertical and horizontal directions, the longitudinal mode of the quadrotor UAV is the same with its transverse mode; (2) without tail rotor and vertical tail, the multirotor yaw aerodynamic moment is minimally influenced by the longitudinal and transverse motions; and (3) the multi-rotor modal oscillation frequency is high, leading to poor manual flight stability and low control accuracy.

\section{Design of Environmental Information Detection System}

The environmental parameter detection system usually selectively sets up a ground detection station at a certain location. The detection station is equipped with the corresponding sensor instruments, and then the obtained data are sent to the PC for analysis and processing by means of network, wireless data transmission and memory card. Shang Chunlin et al. researched on improvement of the environmental data quality; Zhao $\mathrm{Yu}$ et al. designed a monitoring system of roadside air quality in cities; the Institute of Remote Sensing Applications of Chinese Academy of Sciences and the Chinese Academy of Meteorological Sciences jointly designed the space-ground integrated atmospheric environment detection system to acquire atmospheric data; Feng Wei from Jilin University designed a system based on the wireless ethernet for atmospheric environment detection. It is difficult to detect vertical pollution sources and deal with environmental emergencies by using the conventional ground atmospheric detection methods. In recent years, a number of studies have been conducted on the environmental information detection system based on the UAV platform in China. For example, Guo Jian from Tianjin University developed the SIM908-based UAV air quality detection system. ${ }^{[7,8]}$.

\subsection{Design of Hardware Devices and Systems}

The quadrotor UAV changes the rotor speed by the rotation speed of each motor to achieve the change of lift force, thereby controlling the flight attitude and position and enabling hovering detection and small disturbance to the airflow, so it is suitable to be used as a carrier for the atmospheric detection equipment. The quadrotor UAV is characterized by the large load carrying capacity, high flight control accuracy, stable flight attitude, low noise, convenience for carriage and flexible maneuverability. In addition, its flight control is simple and reliable and the fuselage is light and ingenious, so it can take off and land vertically on a very small ground with a low cost of use. The conventional atmospheric detection equipment is large in size and does not have the functions of recording geographic location and meteorological data and real-time information transmission. Therefore, a stable and high-accuracy atmospheric environment parameter detection equipment suitable for the quadrotor UAV must be developed to achieve simultaneous detection of concentration of particulate matter, carbon 
monoxide, carbon dioxide, sulfur dioxide, nitrogen dioxide and other gas concentrations as well as environmental information of pressure, temperature, humidity and geographic location, and support remote data transmission and real-time display functions.

The hardware design of the UAV-based atmospheric environment detection system mainly includes the UAV system and the atmospheric environment data acquisition system. The overall framework of the hardware system is shown in Fig. 2. The small quadrotor flight platform is a non-coaxial multi-rotor aircraft capable of vertical takeoff and landing and autonomous hovering ${ }^{[9,10]}$. F450 aircraft is selected with the maximum takeoff weight of about $2 \mathrm{~kg}$, using 3Sli-Po battery that has an endurance of $10 \mathrm{~min}$. The whole system weighs $1.1 \mathrm{~kg}$, having GPS mode, attitude mode and autonomous cruise mode and supporting the software controlled flight in the ground station. The micro environment detector uses ATmega328 as the core main control board. The sensors used in the measurement mainly comprise a temperature and humidity sensor, a particulate matter sensor, a carbon dioxide sensor, a carbon monoxide sensor and an SD card reading and writing module. The UAV-based atmospheric environment detection system is mainly composed of a UAV system, an atmospheric environment data acquisition system and a data aggregation service center. The UAV system is mainly used to carry the atmospheric environment data acquisition system to the planned region to acquire the atmospheric environment data. The atmospheric environment data acquisition system comprises PM2.5 sensor, $\mathrm{SO}_{2}$ concentration sensor, temperature and humidity sensor and WCDMA (GPRS) wireless data module. Since the UAV system is incapable of carrying too heavy equipment, in order to reduce the weight of the entire system, a minimal system design is adopted to maximize the flight area and flight time of the whole system. The data aggregation service center processes, analyzes and stores the collected data. The ArcGIS Engine service is used to achieve the real-time query analysis, historical retrieval, and plotting of historical air quality curves for a given period of time.

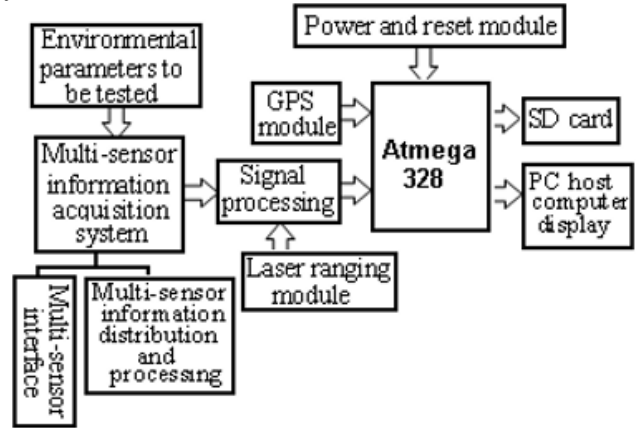

Fig.2 General structure of the system

The multi-sensor information acquisition system software and hardware are divided into four components according to functions. The hardware circuits of the acquisition instrument mainly include power supply circuit, crystal oscillator circuit, microcontroller processing circuit, data acquisition and storage circuit. The acquisition system hardware circuits mainly consist of Arduino UNO development board, power circuit, crystal oscillator circuit, various sensor detection circuits, SD card reading and writing circuits, as shown in Fig.3.

Arduino is a convenient, flexible and easy-to-use open-source electronic prototyping platform. It is based on the open source hardware platform of ATMEL AVR microcontroller and has a special development environment. The Arduino UNO core processing unit ATmega328 contains 14 digital input/output interfaces, 6 PWM outputs, 6 analog outputs, a $16 \mathrm{MHz}$ crystal, a USB interface, an ICSP interface and a reset button. The MICRO SD card reading and writing module can complete the file reading and writing function through the file system and SPI interface driver. The module supply voltage is $4.5-5.5 \mathrm{~V}$, with an onboard $3.3 \mathrm{~V}$ voltage stabilizing circuit, a standard SPI communication interface. There are 6 electrical pins, of which MISO, MOSI, CLK are SPI buses, and CS is chip select connected with the microcontroller unit.

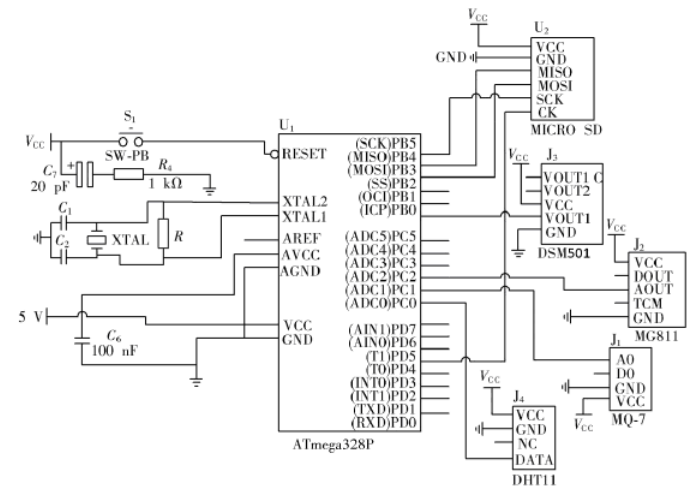

Fig.3 Hardware circuit diagram

\subsection{Environmental Information Detection Experi- ment and Sensor Function Module}

The quadrotor UAV platform carries a camera and an ambient air quality detection pod and hovers above the selected detection points to obtain the gas concentration and other environmental data of the target area.

(1) PM2.5 sensor module: PM2.5 sensor (transmitter) is a digital universal particulate matter concentration sensor based on the principle of laser light scattering. It can continuously collect and calculate the number particulate matters with different particle sizes suspended in the unit volume of air, that is to say, the concentration distribution of particulate matters, which is converted into the mass concentration and output in the form of general-purpose digital interface. The sensor can be embedded in various instruments or environmental improvement devices in respect of concentration of particulate matters suspended in air to provide accurate concentration data in a timely manner. The main technical parameters of PM2.5 sensor: measuring range: 0-1000 ug/m $\mathrm{m}^{3}$, accuracy: $\pm 10 \%$, resolution: $1 \mathrm{ug} / \mathrm{m} 3$, RS485 signal: supporting the ModBus protocol (the baud rate of 9600 and address $0-255$, both of which are settable), and working temperature: $-10^{\circ} \mathrm{C}-65^{\circ} \mathrm{C}$.

(2) DSM501A dust sensor module: The DSM501A sensor, developed by SYHITECH, achieves the quantitative particle density measurement with the principle of light scattering. Particles are heated by the 
heater and move to the LED irradiation area. The different concentrations of particulate matters correspond to different light scattering degrees, and the scattered light is converted into PWM waves by the photoelectric converter through the lens. The main electrical parameters: working environment: $-10^{\circ} \mathrm{C}$ to $60^{\circ} \mathrm{C}$, detection range: $15000 / 283 \mathrm{ml}$, minimum particle detection capacity: $1 \mu \mathrm{m}$, and stabilization time: about 1 minute after the heater power is turned on.

(3) Sulfur dioxide gas sensor module: The intelligent sulfur dioxide $\mathrm{SO}_{2}$ gas sensor is a new type of intelligent sensor specially developed for gas detector manufacturers. It mainly aims at solving the problems of a wide variety of gases to be detected, incompatibility of sensors, complicated production calibration and restriction in the replacement of core components. The main technical parameters of intelligent gas sensor: model $4 \mathrm{NE} / \mathrm{SO}_{2}-20$, detection range $0-20 \mathrm{ppm}$, resolution $0.3 \mathrm{ppm}$, response time $<30 \mathrm{~S}$ and working temperature $20 \sim 70^{\circ} \mathrm{C}$.

(4) Temperature and humidity sensor DHT11 module: DHT11 sensor is a temperature and humidity composite sensor containing calibrated digital signal output. It applies the special digital module acquisition technology and the temperature and humidity sensing technology. DHT11 can measure the real-time ambient temperature and humidity data with the temperature range from $0^{\circ} \mathrm{C}$ to $50^{\circ} \mathrm{C}$ with the detection accuracy of $1{ }^{\circ} \mathrm{C}$; and the humidity detection range ${ }^{[11]}$ from $20 \%$ to $90 \%$ RH with the detection accuracy of $1 \% \mathrm{RH}$.

(5) Carbon dioxide sensor MG811 module: MG811 solid electrolyte sensor is composed of solid electrolyte, a gold electrode, a heater, a platinum lead. It has good sensitivity and selectivity to $\mathrm{CO}_{2}$, and excellent stability and reproducibility slightly affected by changes in temperature and humidity. The module comprises a signal amplification circuit and a temperature compensation circuit. The main technical parameters: heating voltage $6.0 \pm 0.1 \mathrm{~V}$, heating resistance $30.0 \pm 5 \% \Omega$, heating current about $200 \mathrm{~mA}$, working temperature -20$50^{\circ} \mathrm{C}$, output signal $30-50 \mathrm{mV}$ and corresponding to 350 $10000 \mathrm{ppm} \mathrm{CO}_{2}$.

(6) MQ-7 type carbon monoxide sensor module: MQ-7 sensor is a semiconductor gas sensor, which is made by the whole microelectronics technology and has the characteristics of high sensitivity and stable performance. The detection range is $10-1000 \mathrm{ppm}$. It adopts the high- and low-temperature cycle detection method. When heating at a low temperature and $1.5 \mathrm{~V}$, the carbon monoxide is detected. The conductivity of the sensor increases with the increasing concentration of carbon monoxide in air. Stray gases are cleaned at a high temperature (5.0 V heating) and adsorbed at a low temperature. A simple circuit can be used to convert the change of conductivity into the output signal corresponding to the gas concentration.

\subsection{Data Communication Module Design}

Arduino IDE is a software compiler for Arduino development board program that can complete the program compilation, debugging, uploading and other tasks. ArduinoUNO is connected to the computer with USB cable. After the correct matching model, baud rate and other content are selected, the data transmission and receiving status can be displayed in a real-time manner. The environmental parameter acquisition system software is mainly composed of three functional modules. The system is powered on and warmed up to complete the initialization of the sensor and SD card. The temperature, gas, and particulate sensors are used to obtain the data of the current environmental test objects, perform the signal conditioning, transmit them to the Arduino processing unit and output the corresponding analog values of the data. The analog quantity is converted into the corresponding concentration value in combination with the characteristics of the sensors. Arduino processing unit stores these data in the SD card, reads them on the computer and performs subsequent operations such as data visualization, analysis and processing.

The environmental information detection system aims to transmit the collected environmental data to the terminal server platform in a real-time manner. For this purpose, the system uses SIM5320, a WCDMA module launched by SIM, which can run in $3 \mathrm{G} / \mathrm{HSDPA}$ mode and uses the serial transmission mode to transmit data with STM32F103. The standard AT control commands can conveniently realize the functions of call, SMS and data transmission, and thus being suitable for the streamlined development and design. The multi-sensor target location of SIM5320 module and the environmental parameter detection system needs to obtain the geographic location information of the system. The GPS module needs to be configured to send the GPGGA format location information to the processor through the serial port at a rate of $4 \mathrm{~Hz}$. After successful configuration, the processor receives information sent by the GPS module, the sensor information acquisition and the node information are sent to the client for detection control, the processing flow of the basic information acquisition module of the sensor and the flow chart of the GPS module positioning software are shown in Fig.4. The sensor of the environment acquisition module communicates with the processor through I2C bus and the sensor information needs to be read while reading the environmental acquisition module information, as shown in Fig. 5 . 


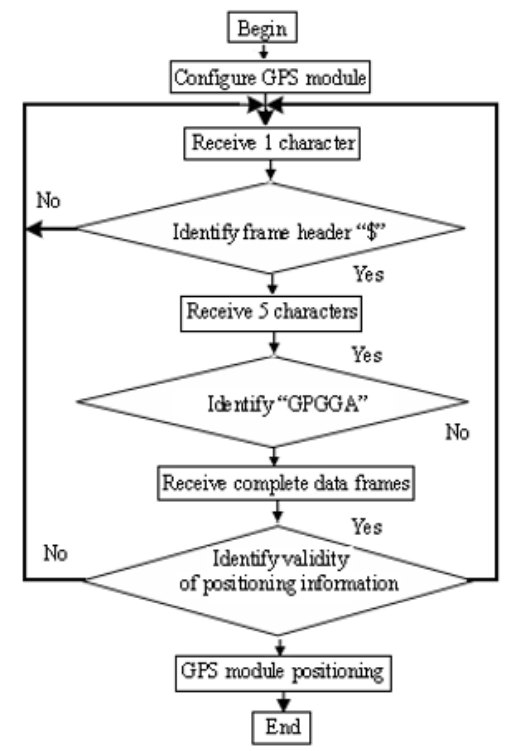

Fig. 4 Flow chart of GPS module positioning software

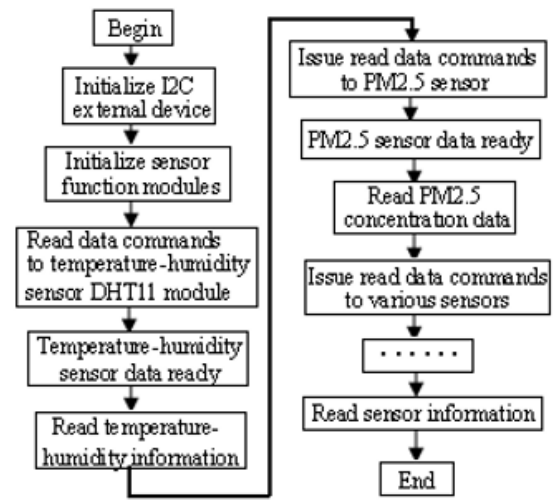

Fig.5 Process of environmental data acquisition module software

\section{Existing Problems and Solutions}

The airflow can influence the quadrotor UAV to a certain degree and the hovering stability needs to be improved. In the light of the timeliness of the real-time monitoring of ambient air pollution, the detection device should be small in size, light in weight and convenient for carriage on the quadrotor UAV platform, so as to ensure the accuracy of measurement results. The numerical simulation methods are used against various pollutions to simulate the environmental conditions of the day of detection, particularly the numerical simulation of wind field. Generally, the quadrotor UAV has a limited load capacity, so efforts should be devoted to the development of high-accuracy and light-weight UAV atmospheric detection pod and the research of environmental quality assessment methods by the atmospheric environment detection system based on the quadrotor UAV platform. This scheme of the air quality monitoring system on the quadrotor UAV platform is generally feasible and requires further study of the following issues: (1) In the initial flight control mathematical modeling, some interference factors are ignored, and the control effect is not sufficiently accurate, so it must be carefully analyzed from the algorithm to enhance the hovering stability. (2) The load capacity and control accuracy of the quadrotor UAV platform must be improved. In terms of hardware devices, especially the selection of motor, control system and battery, better products need to be found. (3) The methods of environmental quality assessment are studied and the monitored areas are analyzed to reasonably plan for the route and identify the task points. The data is received in a real-time manner through WCDMA network. The system is highly stable and can achieve the real-time and accurate monitoring of the atmospheric environmental quality and improve the efficiency of the air quality monitoring system.

\section{Conclusions}

In these years, the demand for remote sensing technology is increasing in many social activities such as environmental monitoring, disasters and resource surveys. The atmospheric environment detection system based on the quadrotor UAV platform is low cost, light, simple and highly extensible. The air data acquisition device with multiple sensor combinations, while ensuring the aircraft reliability, can complete the lowaltitude data acquisition, realize the real-time and quick detection and data acquisition of environmental parameters such as temperature and humidity, PM2.5 concentration, carbon dioxide and carbon monoxide concentrations, and store these data in the SD card for subsequent visualization and analysis. During the experiment, the rotor rotation has a large disturbance to the airflow. The measured results are quite different from the ground observation results. The flight mode has a considerable influence on the measurement. The working time of the system is restricted by the endurance of the quadrotor, so it cannot achieve the long-time acquisition of environmental parameters and the system functions need further optimization.

\section{Acknowledgments:}

We are grateful for the support of the scientific research fund of Yunnan Provincial Department of education

(Project number: 2015Y412)!

We thank the support from Undergraduate Innovation and Entrepreneurship Training Program of Yunnan Province (Project number: 2016A01) !

\section{References}

1. Tao Zhang, Guanjiu Hu, Aiping Deng et al. Discussion on Environmental Monitoring Technical Regulation based on Health Field[J]. The Administration and Technique of Environmental Monitoring, 2016,28(1):1-4.

2. Quanmin, Guo, Xuankun Ma, Jian Wang. Design and Implementation of Indoor Air Quality Detector $[\mathrm{J}]$. Foreign Electronic Measurement Technology, 2015,34(1):52-55. 
3. Qian Liu, Zhihai Liang, Huifang Fan. The Development of UAV Remote Sensing and Its Application in Geo-information Related Industries[J]. Geomatics \& Spatial Information Technology, 2016,39(6):167-169.

4. Zhiqiang Li, Xiaoxu Zhang, Ling Liu et al. Research on UAV Platform for Atmospheric Environmental Monitoring $[\mathrm{J}]$. The Administration and Technique of Environmental Monitoring, 2017, 29(1):65-68.

5. Qingzhan, Zhao, Tianyi Zhang, Hong Chen. Design of air quality monitor for small quadrotor UAV[J]. Modern Electronics Technique, 2017,40(24):94-99.

6. Wei Wang, Lihong $\mathrm{Xu}$, Haigen $\mathrm{Hu}$. Neuron adaptive PID control for greenhouse environment [J].Journal of Industrial and Production Engineering, 2015,32(5):291-297.

7. Nan Zhang. The Design of the Atmospheric Environment Monitoring System Based on ARM[D]. Harbin: Harbin University of Science and Technology, 2014.

8. Jian Guo. Design and research of air quality monitoring system with UAV based on SIM908[D]. Tianjin: Tianjin University.

9. Salih, A.L, Moghavvemi, et al. Flight PID Controller Design for a UAV Quadrotor[J].Scientific Research \& Essays, 2010, 5(23):3660-3667.

10. Yu Song, Junwei Song. Design of micro UAV flight control system based on SOPC[J]. Transducer and Microsystem Technologies, 2016, 35(9):101-103.

11. Mousaad Aly. Atmospheric boundary-layer simulation for the built environment Past, present and future[J]. Building and Environment,2014,(75):206-221. 